\title{
Triggering the Deep Learning Approach in Power System Courses using Free and Open Source Software
}

\author{
L. Vanfretti, Member, IEEE, and F. Milano, Senior Member, IEEE
}

\begin{abstract}
This paper describes how Free and Open Source Software can enable the deep learning approach in power system courses. With this aim, the paper describes authors' experience with PSAT for undergraduate and graduate education. Specific examples of undergraduate activities based on PSAT, such as class activities and course projects, are given as illustrations. Experience with graduate $\mathrm{PhD}$ level education is also described. Interviews with former students reveal the positive impact that the use of FOSS in general, and PSAT in particular, had on their learning and how it has influenced their professional life.
\end{abstract}

Index Terms-Power system analysis, free and opensource software, Matlab, GNU Octave, Python, surface learning, deep learning, functioning knowledge, learning activities, constructive alignment.

\section{INTRODUCTION}

$\mathbf{H}$ OW to motivate students to pursue studies in a specific field has always been a challenge. Another important challenge in technical studies is how educators can teach students more effectively so they can reach the work force with functioning knowledge. These challenges becoming a bigger concern for the power engineering community in Europe and North America due to the eminent aging of the power and energy work force [1], [2].

This paper attempts to give an answer to very general questions arise from the challenges outlined above by focusing on a particular topic, namely, the use of Free and Open Source Software (FOSS) in undergraduate as well as graduate power engineering education. To elaborate an argument responding to these questions, we build on our experience in using and developing a specific FOSS, the Power Systems Analysis Toolbox [3] (as described in [4], [5], [6]), and examine in this article how FOSS can be an enabler of the deep learning approach in power system courses through its use in teaching and learning activities.

There is a strict relationship between enabling deep learning and allowing students to arrive to the work force with functioning knowledge. Of course, not all students are able to reach

Invited Paper, Best Practices in Electrical Power Engineering Education Panel Session, IEEE PES GM 2011.

L. Vanfretti is with the Electric Power Systems Division, School of Electrical Engineering, Royal Institute of Technology (KTH), Teknikringen 33, SE-100 44, Stockholm, Sweden. E-mail: luigiv@kth.se. Luigi Vanfretti is supported by the STandUP for Energy collaboration initiative and the KTH School of Electrical Engineering.

F. Milano is with the Department of Electrical Engineering, University of Castilla-La Mancha, 13071 Ciudad Real, Spain. E-mail: Federico.Milano@uclm.es. Federico Milano is partly supported by the Ministry of Science and Education of Spain through CICYT Project ENE-2009-07685 and by Junta de Comunidades de Castilla - La Mancha through project PCI08-0102.

978-1-4577-1002-5/11/\$26.00 C2011 IEEE the proper maturity to benefit of a true deep learning approach. However, amongst students with potentially promising skills, a good teaching approach can stir the curiosity of the students, and thus, it is more likely that the deep learning is triggered.

The main conclusion to which we have arrived to, after working for almost a decade in power engineering education, is that FOSS is inherently attractive to young people. Most likely because of the idea that FOSS is somewhat "out of the conventional schemes". This notion of "rebellion" is always appealing for new generations. Actually, to this matter, the good point behind FOSS is that being unconventional can be also a positive and productive approach towards society.

PSAT shows students that a free software tool is capable of solving the same problems that costly proprietary software packages solve. Furthermore, PSAT can be opened and the "mystery" behind the internal functioning of a power system package can be unveiled. With sufficient programming skills, PSAT can also be modified, extended and improved. Thus, the students have in their hands a tool that is not only free (i.e., has no monetary cost), but also respects their freedom to create any possible model and solve any problem. Creative students quickly discover through the use of PSAT that power engineering can be actually mastered, and that is closer and more friendly than it may seem at a first approach.

The second author has recently developed the next generation of PSAT by using the Python language [7]. This tool has been used exclusively for research activities and by senior $\mathrm{Ph} . \mathrm{D}$. students. The main goal of this Python project is to ease as much as possible the development of new device models and prototypes of novel algorithms. After a couple of years of development and use of this new tool, the main conclusion that can be drawn is that there is a strict relationship between software's architecture and the triggering of deep learning. In other words, FOSS per se is not enough to stimulate the participation of students in the development of an open source project. The project itself has to be properly structured in order to be easily understood. With this aim, the chosen programming language and software architecture bears a critical role.

PSAT sweeps away the paradigm imposed by monolithic proprietary packages that with complacent prowess are capable of making difficult and unclear that what it is not. This is beneficial for two reasons. Firstly, students learn that even complex software projects such as PSAT can be mastered if properly approached. Secondly, students understand that any software package can be improved, extended and customized. The latter skill is more commonly achieved by Ph.D. students, but it is not uncommon also for motivated undergraduate students to attain it.

We firmly believe that the very mission of the University is not to provide a specific knowledge that will become obsolete 
in a few years or months, but to develop intellectual skills that can be later on applied to any particular practical problem. This is the best functioning knowledge that can be provided to a student, while any other knowledge is ephemeral and fashion prone. The skills to be developed are curiosity, analysis and synthesis ability, and intellectual honesty. With this aim, for the reasons described above, FOSS (and, thus, also PSAT) are efficacious tools.

The objectives of this article are the following:

1) To determine the relationship between FOSS in general, and PSAT in particular, and deep learning in undergraduate courses.

2) To determine the relationship between a "good" open source software architecture and deep learning for $\mathrm{Ph}$.D. research activities.

3) To provide the feedback from former students, now practitioners, that used PSAT while attending power engineering courses.

The remainder of this article is organized as follows. Section II defines surface and deep learning approaches. Section III and Section IV describe our experience with use of PSAT for undergraduate and graduate learning and teaching activities, respectively. Section V provides excerpts from interviews to practitioners that as former students used PSAT in their courses. Finally Section VI draws conclusions and Appendix A depicts the interview that was submitted to former students.

\section{Learning Approaches: Surface And Deep LEARNING}

The concepts of surface and deep learning approaches arise from studies of the contexts in which learning is pursued, that is higher learning institutions, which gave birth to the field of student learning research. Although these concepts are not new and have been properly addressed in the past (see for example the school reform elaborated by Gentile in Italy at the beginning of the XX century [8], [9]); the terms surface and deep learning, as used in Constructive Alignment (CA), theory originated in Sweden [10], [11] in the 70's.

As the term suggest, the surface learning approach occurs when students focus only on covering the mere superficial layer of a course content. The term was coined by Marton and Säljö who noticed two kind of responses from students after assessing a reading assignment [10], [11]. The response from those using the surface approach was characterized by a strong focus on memorization of facts and details without joining these pieces of information together. Although these students had a recollection of terms and isolated items, they did not show an overall understanding of the underlying ideas conveyed in the reading assignment.

There are several factors triggering a surface or deep learning approach in student learning [12], the most important one being the lack of alignment between the intended learning outcomes (ILOs), the teaching and learning activities (TLAs), and the course assessment and grading. Below we only briefly outline some of these aspects. The interested reader is invited to refer to [12] as a starting point for further study.

\section{A. The Surface Learning Approach}

This approach is characterized by a deliberate need to get the course tasks out of the way while giving an appearance of complying with the course requirements, the "principle of least effort" in action. Memorization is a surface approach used to counterfeit understanding. Selected content is learned by rote, leaving a poorly structured knowledge that is not capable of withstanding the test of time.

Nevertheless, the surface learning approach is not a conceived student attitude, it is actually the response of the student to the teaching and assessment conditions. As pointed out by Gibbs \& Tang ( [13]), it is possible to say that "under current conditions of teaching or assessment, he (the student) chooses to use the surface approach". When the assessment system is not aligned with the learning objectives and the teaching activities the students are forestalled to see the structure and significance of what is taught. Hence, it is possible to satisfy the course requirements, and even to get a good grade [14], [15], if the assessment system remunerates rote learning. However, the results from examinations will not guarantee any retention and actual learning.

\section{B. The Deep Learning Approach}

In this approach, students attach value and meaning to their learning process. Motivated by a "need to know", the students undertake the different course tasks seriously. Because of this commitment the students learn the details associated in the learning tasks, as well as understanding the ideas behind the details. As a consequence, a solid knowledge foundation is established allowing students to also understand the "big picture", thus deriving satisfaction and positive feelings. It is interesting to note that this approach is implicit in the Liceo Classico and University courses elaborated by Gentile in [8]. In such an educational scheme, surface learning is futile for passing examinations since mere memorization is by far insufficient to attain satisfactory marks. However, the main drawback of Gentile's approach is that students that have no natural disposition for the deep learning typically abandon their studies.

Nevertheless, the student factors mentioned above are not independent of the teaching strategies, the deep approach will not be triggered if the course ILOs, TLAs and assessment are not aligned. Proper design of each of these factors will aid the students in adopting a deep approach. It is highlighted in [16] that students are most driven to the deep approach when "the route to understanding is through application". In the remainder of this paper we discuss how different TLAs can be designed with the aid of FOSS to trigger the deep learning approach.

\section{A word of caution}

It is worth observing that one learning approach is not exclusive of the other, this means that a student can use both a deep and surface approach in the same course [16]. The culprit for this attitude lies not in the student itself, but most likely the course design, and particularly the course assessment. While a course project or its assignments can trigger the deep learning 
approach, the approach taken for an examination will likely bee surface if no changes to traditional assessment are made.

Nevertheless, as it will be shown latter in this article, and also from evidence in [14], [15], the deep approach results in long-term functioning knowledge and retention. However, the impact of this activities is not fully realized if the entire course assessment is not modified in the lines of CA [12], as it will be discussed latter. This evidence should be taken by educators as a plea for a change in the standard practice of engineering education.

\section{TRIgGering DeEP LeARning In UndergRaduAte COURSES}

This section discusses the design and use of learning activities that, through the utilization of FOSS, trigger the deep learning approach and therefore enable functioning knowledge. Although the learning activities were designed by the authors without previous knowledge of CA theory [12], it is now acknowledged in this article that this theory and the recommendations in [16] are impregnated in the design of the learning activity and the assessment task as described below.

\section{A. Preparatory Learning Activities}

The project was used both as learning activity and an assessment task, preparation for this course project gave students aid in getting started with their course work. To this aim several activities before the course project prepared the students on two fronts: directing them on the theory that they should understand from the course, and building competence on use of the software tool. The preliminary activities to the project are:

1) An informative session on using the FOSS PSAT for power flow analysis was carried out [17].

2) A learning activity focusing on power flow analysis as described in [4] (see Section V) was carried out after the informative session above.

3) A homework assignment on power flow analysis using "paper and pencil" with optional use of PSAT [18].

Note that TLA (1) focuses in building declarative knowledge, while (2) and (3) provided opportunities for functioning knowledge at the relational level. Functioning knowledge at the extended abstract level [12] was achieved through the project described below.

It is important to highlight that the simple class activity of item (2) is most likely only possible due to the open source nature of PSAT. In contrast to commonly used commercial proprietary software used for power system analysis, the possibility of changing the source code of a software is only available in FOSS.

\section{B. Project Design and Elements for Functioning Knowledge that Trigger the Deep Learning Approach}

1) Design and Elements: Teaching is about providing students with the opportunity to learn appropriately and not by resorting to shortcuts, this can only be accomplished by encouraging them to adopt the deep learning approach. The course project proposed to the students is outlined in Appendix $\mathrm{A}$, it is a case-based learning activity with group work format.
The ILOs of many engineering courses stress an importance of using applying knowledge in practical contexts, yet the traditional course assignments provide little opportunity for this [12]. The first step is to build a declarative knowledge base (as described in the previous section) that can be exploited to apply their knowledge to make informed decisions. The next step is to put this knowledge into action, as in the proposed project.

The project considered real-life design constraints and limitations, and the students where asked to select one of the designs that was the better suited for the design specifications based on an technical-economical engineering judgment. ${ }^{1}$ The goal of the project is to trigger the deep learning approach at the different cognitive levels of the SOLO taxonomy [19], while putting the emphasis on the higher cognitive levels. To this aim "Suggested Project Tasks and Guidelines" encouraged the students on the three highest levels of the SOLO taxonomy, the active verbs from the taxonomy have been underlined below:

1) Quantitative Multistructural Level: students where asked to compute a load flow and describe the operational issues that the system presented.

2) Qualitative Relational Level: students where asked to analyze the condition of the system, to explain why this condition existed and why it was unacceptable. The students later applied their knowledge on power system analysis techniques to their solutions.

3) Extended Abstract Level: the students where asked to hypothesize on possible design solutions, and to test each of them. They were then asked to reflect on their designs, to judge which solution was best under the given design constraints, and to argue their choice based on their knowledge and previous analyses.

This approach puts forward several important elements that encourage deep learning:

- It sets criteria demanding learning at the highest cognitive levels, particularly at the extended abstract level [19].

- Promotes a divergent ability [12], i.e. it generates alternatives that make way for other assessments of value such as originality, and creativity.

- Promotes reflective learning, the guidelines and suggested tasks provided students with a series of questions triggering reflection.

- It uses simulations that allow students to adapt parameters and perform changes so to answer "what would happen if?" questions.

- It provides a context where unintended learning outcomes can flourish.

- Promotes the use of FOSS, enabling the students to explore an unbounded set of possibilities, and respecting their freedom to explore.

${ }^{1}$ While this project used power flow simulations, its main aim was not to help the students to learn about power flow, but to apply their knowledge in power system analysis. More complex design techniques can be also achieved by using complementary analysis methodologies such as contingency analysis, off-line security assessment, etc.; when using these alternative methods other important underlaying concepts that are difficult to learn can also be revealed. See the quotes from students comments in p.4. 
Below we highlight interesting quotes from student projects, these quotes reflect that the project design and the availability of a FOSS for power system analysis were enablers for their learning and for carrying out their project. Firstly, one of the student groups recognized the challenges in achieving high level cognitive demands, here the FOSS PSAT acted as an enabling tool allowing to reach the project goals:

The most difficult part of this project was comprehending what was happening in the system and how to use our knowledge of power systems and the software to find a practical solution.

Moreover, it is important to recognize that this project provided a learning context that enabled unanticipated learning outcomes, from the conclusions of one of the projects we quote:

Although we eliminated all of the system violations and corrected the bus voltages to within the desired $5 \%$ deviation ${ }^{\alpha}$, another problem still remains $\ldots{ }^{\beta}$ However, it would be extremely wise to construct several new transmission lines in order to maintain overall stability to the system. This would provide more reliable service to the system and eliminate the potential for a costly and perhaps catastrophic fault.

In this excerpt we observed that in $\alpha$ the students satisfied the design criteria, they could have stopped at this point because all the requirements for the project were satisfied, but they were in 'need to know' and in $\beta$ identified additional issues with their design. As an unintended learning outcome the students applied their knowledge on security and stability assessment by elaborating a third solution which also addressed these additional technical issues (in $\gamma$ above). This learning opportunity would probably not be available in a course project design that does not encourage the deep learning approach.

\section{Assessment and Grading for Functioning Knowledge}

Assessment involves a judgment on how well the design accounted with the constraints and limits expressed in the project description. The important factor here is that the student shows an understanding of near-real-life limitations and how the problem can be reasonably attacked. This is why an open-ended assessment was chosen.

In assessing three stages where used: (i) criteria for assessment was set, (ii) the students selected evidence to be judged against the criteria, (iii) a judgment was made about the extent if the criteria was satisfied. The assessment format for this project was through a group project, while the grading for was performed using a set of high level criteria (see Appendix A), instead of the methods used in summative assessment which reward marks to each of the completed tasks. Evidence was selected by the students and a reflective group report submitted by the groups of students was read, a group grade was issued to the students. Although an individual grade could have been determined for each student based on their individual reflective reported efforts, it was found that the students worked cooperatively during the whole project, and thus this was unnecessary. The reflective reports showed that they had applied the knowledge acquired in the course and that the deep learning approach was triggered as discussed in the previous Section. Because of the value that the students attached to complete this project, there were no incidents requiring re-grading and most students were satisfied with their grade, note that most of the students ( 8 out of 10) deserved and were awarded the highest mark on the project.
Nevertheless, this learning activity and its assessment were not a determining factor of the final grade of the course. Because of this it is likely that the students undertook both the surface approach (for the final examination particularly) and the deep approach (for the course project) as their strategy for dealing with the course.

The student interviews reported in Section V reveal that despite this drawback the course project using PSAT allowed the deep learning approach to enable long-term functioning knowledge. However, to fully exploit these benefits at the full extent it will be necessary in future occasions that CA is used for course design.

\section{TRiggering DeEP LeARning in Research ACTIVITIES}

PSAT has been available on-line since January 2003. Since then, the contributions in terms of new device models and algorithms has been very scarce. This is uncommon in most open source projects which typically take advantage of user contributions for quick development and extension of the original code. An analysis of the reasons for this failure of the PSAT as a FOSS can be found in [6].

The meager participation to the development of PSAT implies that while the community of PSAT users is relatively huge, the community of PSAT developers is limited to very few people, generally working in collaboration with the principal PSAT developer. Strictly related to the clear difficulty in creating a community of PSAT developers is the difficulty of getting into the PSAT code. In fact, the current publicly available version of PSAT has not been planned for being "thought", but, rather, for being developed by one person. This is due to the fact that the gross part of PSAT has been made available when all main choices regarding the software architecture were already taken.

Based on this experience, the second author of this article has developed a new software package, mainly from scratch, but constantly looking at the legacy of PSAT. Experience both as developer and as discloser of the software architecture and supervisor of Ph.D. students has been taken into account. The main objectives kept in mind when developing this new project were the following:

1) Use an architecture flexible enough to cover any possible power system device model and solver algorithm.

2) Use of a sufficiently modular structure to allow a concurrent utilization by the main developer as well as by a variety of other researchers and students.

3) Use of a hierarchical, class-based organization of device models that allows also a non-expert user (e.g., undergraduate students working on their final project) to be able to write their own code that seamlessly integrates in the main project distribution.

After a careful and farsighted reflection, it was sufficiently clear that these goals could not be obtained simply rewriting some parts of PSAT. The main issue was the language itself, i.e., Matlab. Paradoxically, the most commonly used scientific-oriented computer language was actually a strong limitation to the deep learning of the PSAT architecture. This 
does not mean that the MATLAB language is not "good". The MATLAB language has undoubted merits and its diffusion all around the world is a sufficient proof of that. However, the MATLAB language lacks or makes it difficult to take advantage of some basic features of modern object-oriented, high-level computer languages. Without entering into the details, which are out the scope of this paper, it suffices to cite dynamic typing, meta-programming, introspection, polymorphism, etc. Further insights on computer language semantic can be found, for example, in [20].

A natural consequence of the limitations of MATLAB as a computer language, is that MATLAB becomes intrinsically "difficult" to understand and to use for a complex software project. Again, we do not want to convey that the MATLAB language cannot be used of complex projects. Actually, there is a huge variety of complex projects based on MATLAB. Rather, we state that MATLAB might not be a good choice if one wants to develop a complex software project and at the same time has to explain the architecture of such project to others, such as students that are not necessarily skilled programmers and have not the time to master computer programming techniques before approaching such complex software project.

The main point of the discussion above is that if one wants to use a project oriented to power system analysis as a main board for research, such project has to be easily understood. On the other hand, simplicity should not result in reducing or limiting the versatility, and the generality of the project itself. It appears that an adequate solution, able to accommodate these two apparently incompatible requisites, is to organize the project as a layered and modular software architecture.

The top layer is the one that an end user is going to use (e.g., user interface). Then a second layer is composed of device models. Users interested in modifying or adding a new device do not need to know anything but the general device structure. In other words, the user is not forced to know lowlevel device functions, but only top-level ones. This can be easily obtained using classes and meta-programming. A deeper layer is the one implementing routines that handle devices, such the time domain integration. A user that wants to define a new algorithm for time domain integration has not only to understand the basic functioning of each device, but also how devices interacts with the whole system. Writing a new algorithm requires a deeper understanding of the code, but it is also relatively less common than the need of implementing a new device model.

Figure 1 illustrates the organization of a power system software project in layers. The figure shows that there is still a need for a project maintainer that knows all the details of the project, but this is the common situation of most FOSS projects. We want to stress the attention on the fact that the layer approach is also an indicator of the level of the surface/deep learning of the user. As indicated in Section II, these approaches are not mutually exclusive but may always coexist. As a matter of fact, the same main developer is a surface user when running the program user interface.

The main issue of the MATLAB-based version of PSAT has been, and currently is, to mix together all layers, especially those regarding device modeling. As it is clear, for the main

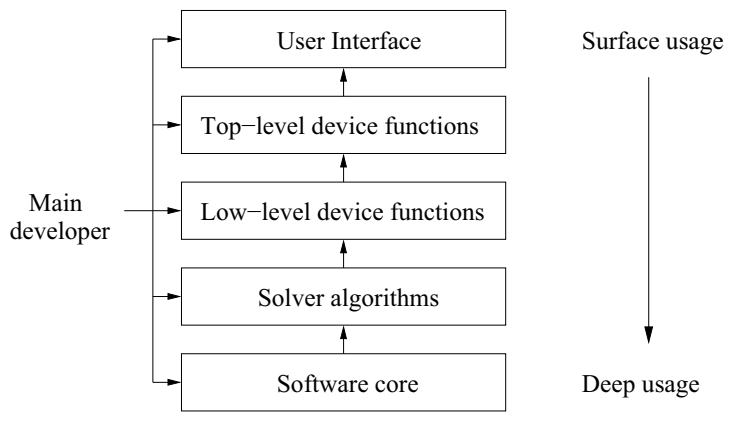

Fig. 1. Layer organization of a FOSS power system software project oriented to teaching and research.

developer mixing everything together is not an issue, since, the main developer has to know everything in any case. However, if one needs to explain the code to others, the organization in layers holds a key role.

The need of meta-programming and a strong class-oriented programming led to the risky choice of abandoning MATLAB and adopting Python [7], [21]. The interested reader can find a very interesting discussion on the advantages of this language for general-purpose scientific programming in [22], while an extensive monograph on the organization and the results obtained with of the new Python-based project can be found in [23].

For the scope of this paper, we limit to report the results of using the new Python-based project for research activities. The software has been explained to two visiting Ph.D. students at the University of Castilla-La Mancha (UCLM). The visits were oriented to the development of new models of wind turbines (in particular, direct drive synchronous machines, DDSG) and photo-voltaic (PV) cells, respectively. The time of the visit was limited to three months, so there was a need to reduce the learning step in order to begin as soon as possible the implementation phase. The results were unexpectedly positive. Both students did not know Python before arriving at the UCLM, but were able in just three weeks to start implementing the new models. At the end of the visiting periods, 5 DDSG and 6 PV cells models as well 2 sun irradiation models have been fully implemented and tested. In both cases, the devices implemented include tens of state and algebraic variables, complex nonlinearities involving variable hard limits and intrinsic modeling complexity. Even though these students demonstrate quite exceptional skills and a clear deep learning attitude, these results could not have been possible using the MATLAB-based version of PSAT.

\section{Student Interviews}

\section{A. Approach and Design of the Student Interviews}

Three out of ten students from the course "EPOW 4010 Power Engineering Fundamentals" taught during Fall 2006 at Rensselaer Polytechnic Institute, Troy, NY, were contacted to fill out the interview given in Appendix B. Performing these interviews three years after the students took the course and now that they are part of the professional work force might be seen as unconventional, but the authors believe that this approach allows to determine if deep learning was triggered hence enabling long-term functioning knowledge. 
Nevertheless, the drawback of this approach is that some of the students did not have a complete recollection of some of the details the authors were probing. It is recommended that those attempting a similar study carry out two sets of interviews, one immediately after the course has been completed. The first interview can be recorded and transcripts can be saved to provide materials for analysis. A second interview after the students have graduated and are part of the active work force (as done in this article) can be most helpful in determining if functioning knowledge was achieved through learning activities that trigger the deep learning approach.

The questions from the interviews given in Appendix B have a heading in boldface indicating what was being probed. This headings were not distributed to the students, but are included here so that it is possible to correlate the critical analysis given below. The questions focus only on the course project which used PSAT as their main tool, and not the course as a whole allowing analysis of the impact of this FOSS in their learning.

Differently from the standard practice of using Multiple Choice Questions (MCQs), student interviews where formulated using open-ended questions requiring the students to write down small sentences (or as much as desired). The only drawback from this approach is that no statistical analysis can be performed as when MCQs are used [5]. However, taking into account that the course had a small population, the authors believe the statistics in this case might not be as meaningful as the analysis from student feedback.

\section{B. Analysis of interviews}

Responses of four students to the questionnaire in Appendix A where compiled and analyzed. Because of the amount of feedback, we only provide analysis and excerpts to some of the students' answers. Additional analysis will be made available in future publications.

The overall impression of the students of the project was quite positive, it was found by interviewing the students that the approach used in the project design effectively enacted on the recommendations in [16], creating a route to understanding through application which prepared students for engineering practice. This is highlighted by the responses of the students to Q.1:

It was nice to work on a project similar to what you would be working on when in the work force. While in school you tend to do a lot of smaller problems by hand, but in reality, you would be looking at a power system in whole and seeing the effects of the changes. It was good to replicate that with the project.

Another similar quote of a student's response to Q.1 was:

This was one of the more interesting projects that $i$ had as an undergraduate, it had a clear goal but very open-ended ways to qchieve that goal, giving us the freedom to simply experiment with the system and learn how a real power system would work.

The effect on functioning knowledge for professional practice of the project design approach should not be underestimated, this is clear by the response to Q.2 by one of the students:

In the work force everything can be open-ended. The customer is relying on your recommendation based on the study. So open-ended problems, although uncomfortable, are good for practical problems.

More importantly, an illuminating response to Q.18 highlights how the course project using PSAT allowed the students to engage in deep learning and how it enabled their long-term functioning knowledge:
My current job is basically a much larger-scale version of the course project, as we are examining deficiencies in the transmission system and trying to find cost-effective ways to cure these problems.

To support this evidence an answers to Q.19 show how through the course project using PSAT it was possible to lay a strong foundation of long-term functioning knowledge:

The project did a good job of showing the basic concepts of power engineering, which enabled me to start my jog with a good knowledge of the conceptual workings of a power system. This has let me quickly expand these concepts to a larger power system, and get a good intuitive feel for how the power system will function with a number of different upgrades added to the system.

Having the FOSS PSAT allowed students to simplify their work flow, the user friendliness (described in detail in [6]) is an enabling factor that other packages don't offer. This is reflected by the student when responding to Q.8:

Made things easy. When justifying a suggestion all you had to do was plug in the parameters, run the program, and explain the results.

A similar supporting answer is given by another student to Q.3:

It was much easier to use (as a begginer) than many other proprietary power flow programs I have used since, especially on a simple system (such as the one used in the project).

More importantly, while it might appear surprising to the reader, FOSS projects such as PSAT are usually of of high quality compared to proprietary software. This is evidenced by an answer to Q.20:

The user interface was easy to learn and made it easy to quickly apply different solution ideas to the power system. Without this, textbased programs (including some of the proprietary programs which I use in industry today) make it much harder to quickly test and evaluate different solutions, and more difficult to see how power flows are distributed across the system

When asked Q.24, regarding if there should be changes to the project, one of the former students replied:

No changes. Just add one or two more smaller exercises during the semester. The students will probably complain, but who cares what they think. They'll thank you later.

The questioned student is referring to "Preparatory Learning Activities" in Section III-A. It is interesting to note that the student conveys that even though students might "complain" about having more exercises in class, it will be beneficial in the long-term. It is quite unusual that a former student asks for more learning activities, thus proving the positive feelings the learning activities generated.

\section{CONCLUSIONS}

This paper has shown that FOSS has a great potential to support teaching and learning activities for undergraduate education, and research training at the graduate level. The use of PSAT aided in triggering the deep learning approach at the undergraduate level through class activities by allowing students to ask "what if?" questions by freely modifying the source code in the software, this would have been impossible without a FOSS. In addition, students were free to explore different alternatives for the designs in their project course thanks for the features of PSAT as an educational FOSS [6].

Although the accomplishments reported in this paper are encouraging, the authors wish to raise a voice of concern: despite the many efforts [4], [5], [6] for enhancing education through FOSS, it is expected that the massive adoption of FOSS for education in power engineering will continue to be a failure [23]. An educational change is required so 
that engineering students are taught to question commonly accepted assumptions and simplifications, and are motivated to understand the intriguing mechanisms between theory, modeling, and scripting. This educational change will not take place if the appropriate tools for teaching are not embraced, currently education is carried out with restrictive proprietary software tools that do not allow students to explore. To add to that, the common surface learning approach is triggered by conventional teaching and assessment mechanisms that deprive the students from the possibility of awakening their "need to know".

We therefore hypothesize, that this educational change can only be brought upon through the implementation of teaching and learning methods from constructive alignment theory were the deep learning approach is triggered through the use of FOSS providing students with the freedom to learn and explore, a freedom that is commonly denied by opaque proprietary software applications.

Finally, we see that there is the need of FOSS projects specifically designed to be "good" deep learning trigger. The layer approach adopted for the next generation of Pythonbased PSAT project appears to be be very promising in this direction. However, much work has still to be done in this field.

\section{ACKNOWLEDGMENTS}

The authors would like to thank Mr. Joseph (Joe) Crispino, Mr. Dan Schwarting, and Mr. Tom Fredeman, for their feedback provided in the student interviews. The second author would also like to thank Dr. Giuseppe Forte and Dr. Alessia Cagnano for being the first developers of the Python-based PSAT project and for their excellent work at UCLM.

\section{APPENDIX A \\ Course Project Description}

\section{Introduction}

In this project you will use your knowledge of electric power transmission, power engineering tools such as load flow analysis and the PSAT to analyze the current status of the Modified IEEE 14 Bus test network and to propose solutions for normal operation of the network. Due to maintenance and insulation upgrading some of the transmission lines of the system are out of service. Also, the load of the system has changed due to an increase of air conditioning loads. This results in the modified version of the IEEE 14 Bus system shown in Figure 1 (not included here), you do not need to set up the system in PSAT, you will be provided with the PSAT-Simulink model for your convenience.

\section{Design Objective}

To propose and implement possible solutions to establish a normal operation of the system with a voltage deviation of $5 \%$ at all the buses, line loading below $100 \%$ in all the transmission lines and no other limit violations in the network for all the scenarios of the study.

\section{Suggested Project Tasks and Guidelines}

The following tasks and guidelines will help you carrying out your project. While the only mandatory part to submit is Part III, the guidelines in Part I and Part II will give you a good framework to develop your project.

\section{Part I:}

1. Obtain the power flow solution for the initial conditions of the power system

2. Analyze the line loading of each transmission line with the results obtained with the power flow solution. (To check if any line is violating its maximum capacity click on the "Check limit violations" option before you produce a power flow report)

3. Compute the total active and reactive power losses.

4. Obtain a voltage profile plot of the bus voltages.

5. Please describe what are the violations to the system operation.

Once the tasks described above have been carried out, answer the following questions making reference to the names or numbers of the buses or other elements of the power system:

a. Why is the current state of the system not acceptable for reliable operation?

$b$. From the power flow analysis: what abnormalities do you observe on the operation of the power system?

$c$. Regarding the former question: how do these abnormalities affect the satisfactory operation of the system?

$d$. What are your proposals to eradicate the existing abnormalities on the operation of the system? (Provide at least 2 alternatives)

$e$. Please do a technical-economical analysis of each of the proposals you have provided above. (This means you have to consider which of your options is the most economical while still satisfying the technical constraints making this the best choice. Note that you do not need to give actual real costs, you should rather do a qualitative comparison using your best engineering judgment.)

$f$. Select the most adequate and feasible option from the ones you have provided above and justify your choice from a technical-economical standpoint.

Part II:

For the alternative selected from your analysis of part one, please do the following: 1. Simulate the scenario of the alternative you have selected making all the changes necessary to the power network (adding lines, adding shunt capacitors, etc.)

2. Repeat all the numbered steps of part one (one to four)

3. Provide comparative tables for the operating scenarios with and without the alternative you have implemented.

4. Do the system operating conditions improve? If they improve, how good is the improvement? (You have to satisfy the design criteria of voltage deviation of $5 \%$ at all the buses, line loading below $100 \%$ at all the lines and no other limit violations?) 5. Regarding the former step: are the objectives of the project satisfied?

6. If the design criteria are not satisfied you will have to select a new alternative to improve the operation of the system and repeat all of the above.

\section{Part III:}

This part is concern with a report of your findings of parts one and two. Your report must include the following elements, note that there is no template or required format, however the elements below should be present in some form in the report.

$\rightarrow$ A brief introduction summarizing the contents of your report.

$\rightarrow$ A description of your design methodology.

$\rightarrow$ Present the technical-economical evaluations that led to your design (you must provide at least 2 )

$\rightarrow$ Present your comparative results (from task 3 of part 2) (Graphs and Tables are strongly suggested)

$\rightarrow$ A conclusion summarizing your findings and justifying your design from a technical-economical standpoint

Note that presentation and neatness will be taken into account in your grade.

\section{Group Members}

You are required to make groups of at least two students and maximum three student and work as a cohesive unit for the development of the project. When you hand in your project you must submit and additional page stating the contribution of each group member.

\section{Grading}

None of the tasks will be graded independently and no summative grade will be given for all of the individual tasks. Your project will be graded with the following criteria: i) evidence of the application of your power engineering knowledge, ii) the methodology used for your study, iii) the solutions you designed, and iv) your technical and economical arguments of the selected solution. An overall project grade will be given based on the grader's assessment of these criteria.

The project will be given an overall group grade that reflects the group effort to do the assignment. Additionally an individual grade will be given to each student for his personal contribution to the project.

\section{APPENDIX B}

\section{QUESTIONNAIRE FOR THE STUDENT INTERVIEWS}

\section{Teaching for Creativity}

1. Was there anything different in this course project to other course project you carried out during your undergraduate studies?

2. This project had only two binding requirements, and provided a guide on how to perform the different analyses. Do you think having these kind of open-ended problems was positive or negative for your learning?

3. Thinking about what you had to do to complete the project, was the open-source features of PSAT something that facilitated you in performing the project?

4. Did the open-source nature of PSAT enabled you in searching for the different options for your design?

5. Did PSAT gave you the freedom you needed to ask "what would happen if?" questions when you where doing your designs?

\section{Reflective Learning}

6. The project faced you with a power system with unacceptable operation and many 
challenges. While doing your different designs and selecting the final option, can you mention if you had any evaluation assessment to check your assumptions and the solutions you where proposing?

\section{Effect of Working in Groups}

7. You worked in your project with a partner. While coming with different designs for the project, did the fact that you had a partner helped you in coming up with different solutions?

8. Did using PSAT (i.e., having the same open tool) had an impact on working with your partner? Did it make things easy or difficult? Please explain.

\section{Intended Learning Outcomes}

9. The course teaches ... Do you think this course project helped you in achieving the objectives of the course?

10. Please explain if the use of PSAT for the course project aided in this matter?

\section{Teaching/learning activities for apply}

11. While doing your project, did you found yourself using the concepts of power flow given in class so to perform your analysis and designs?

12. Is there any particular example you can point to from the course project?

13. Do you think PSAT had any particular features that let you apply your knowledge effectively?

\section{Unintended Learning Outcomes}

14. Did you learn additional topics than those covered in the course through completing the course project?

15. Did using PSAT also helped you in learning additional topics from other fields or developing other skills than those intended in the course covered in the course?

\section{Long-term Functioning Knowledge}

16. Where do you work now and what is your "title"?

17. What kind of activities do you carry out in your "title"?

18. Do you think the course project helped you in learning the topics that you need as a basis for your current job?

19. Have you found that the activities you had to perform in the project prepared you for dealing with more complex systems as those seen in your current work?

20. Thinking about the way you used PSAT, is there any particular characteristic that enabled you to do your course project that you found useful?

21. Which features from the above would you like to use to perform power system analysis (or any other analysis and design) tasks in your current work?

\section{Additional Feedback}

22. Do you feel you got enough preparation on the use of PSAT to carry out the project? If not, was it easy or difficult to find your way around PSAT?

24. If this project were to be proposed again, what changes would you suggest on the description itself?

25. Did you got appropriate and timely feedback from the teaching assistant? What other things could have the teaching assistant do to help you?

Comments

Please add any additional feedback that you would like to give.

\section{REFERENCES}

[1] J. Giri, "Industry perspectives on university research programs," in IEEE/PES Power Systems Conference and Exposition, 2009, pp. $1-1$.

[2] G. F. Reed and W. E. Stanchina, "The power and energy initiative at the university of pittsburgh: Addressing the aging workforce issue through innovative education, collaborative research, and industry partnerships,' in IEEE PES Transmission and Distribution Conference and Exposition, 2010, pp. $1-7$.

[3] F. Milano, "An Open Source Power System Analysis Toolbox," IEEE Transactions on Power Systems, vol. 20, no. 3, pp. 1199 - 1206, Aug. 2005.

[4] L. Vanfretti and F. Milano, "Application of the PSAT and Open Source Software for Educational and Research Purposes," in IEEE Power \& Energy Society General Meeting, June 2011, pp. 1-7.

[5] F. Milano, L. Vanfretti, and J. C. Morataya, "An Open Source Power System Virtual Laboratory: The PSAT Case and Experience," IEEE Transactions on Education, vol. 51, no. 1, pp. 17-23, February 2008.
[6] L. Vanfretti and F. Milano, "The Experience of PSAT as a Free and Open Source Software for Power System Education and Research," International Journal of Electrical Engineering Education, vol. 27, no. 1, January 2010.

[7] G. Van Rossum, "Python programming language," available at: www.python.org.

[8] G. Gentile, La Riforma dell'Educazione. Bari: Gius Laterza, 1920.

[9] _ "Il Concetto Moderno della Scienza e il Problema Universitario," Libreria di Cultura, 1921.

[10] F. Marton and R. Säljö, "On qualitative differences in learning i: outcome and process," British Journal of Educational Psychology, vol. 46, no. 1, pp. 4-11, 1976.

[11] — "On qualitative differences in learning - i: outcome as a function of the learner's conception of the task," British Journal of Educational Psychology, vol. 46, no. 1, pp. 115-127, 1976.

[12] J. Biggs and C. Tang, Teaching for Quality Learning at University, 3rd ed. Berkshire, England: Open University Press, 2007.

[13] G. Gibbs, Assessment matters in higher education: choosing and using diverse approaches. Open University Press, February 1999, ch. Using Assessment Strategically to Change the Way Students Learn, pp. 41-53.

[14] K. Edström, J. Törnevik, M. Engström, and A. Wiklund, "Experiences of Learning — Student Accounts Linked with Theory," The Wallenberg CDIO Program, Royal Institute of Technology (KTH), Stockholm, Sweden, Tech. Rep., 2001, available on-line: http://www.cdio.org/files/exp_of learning.pdf.

[15] — , "Studentens upplevelser av civilingenjörsutbildningen," Royal Institute of Technology (KTH), Stockholm, Sweden, Tech. Rep., 2003.

[16] _ "Student involvement in principled change: Understanding the student experience," in 11th Improving Student Learning, OCSLD, Oxford, UK, 2003

[17] L. Vanfretti, "A Brief Introduction to the Power System Analysis Toolbox (PSAT) for Power Systems Undergraduate Courses," November 2006, available online: http://idisk.me.com/vanfretti/Public/presentations/ 2006_LV_BriefPSATTutorial_2006.pdf.

[18] _ "Power Engineering Fundamentals - Teaching and Learning Activities Using PSAT," November 2010, available on-line: http://idisk.me.com/vanfretti/Public/pdfs/2010_LV_PEF_Compilation.pdf

[19] J. B. Biggs and K. F. Collis, Evaluating the Quality of Learning: The SOLO Taxonomy (structure of the observed learning outcome). New York: Academic Press, 1982.

[20] B. Pierce, Types and Programming Languages. Cambridge, MA: MIT Press, 2002.

[21] A. Martelli, Python in a Nutshell. Sebastopol, CA: O’Reilly, 2006, second edition.

[22] H. P. Langtangen, Python Scripting for Computational Science. Heidelberg: Springer-Verlag, 2002, third edition.

[23] F. Milano, Power System Modelling and Scripting. London: SpringerVerlag London Limited, 2010.

Luigi Vanfretti (Student Member '03, M'10) is an Assistant Professor a the Electric Power Systems Division, School of Electrical Engineering, KTH Royal Institute of Technology, Stockholm, Sweden, where he was a PostDoctoral Research Fellow during May 2010.

He obtained the Electrical Engineering Degree (with a Power Engineering concentration) from Universidad de San Carlos de Guatemala in June 2005 and received the Francisco Vela award for outstanding academic achievement and best undergraduate thesis. During Fall 2005, he was a visiting researche at the Department of Electronics and Electrical Engineering of The University of Glasgow, Scotland.

He received his MS in 2007 and $\mathrm{PhD}$ in 2009, both in Electric Power Engineering, from the Electrical, Computer, and Systems Engineering (ECSE) Department at Rensselaer Polytechnic Institute (RPI). During Spring 2010 he was a Post-Doctoral Research Associate at RPI working on synchrophasor applications. He was awarded the Charles M. Close Doctoral Prize from RP in May, 2010 for his research and teaching work at ECSE.

His research interests are modeling, dynamics, stability and control of power systems; applications of PMU data for Smart Transmission Grids, and open source software for power system engineering education.

Federico Milano (Senior Member '09) received from the University of Genoa, Italy, the Electrical Engineering degree and the Ph.D. degree in 1999 and 2003 respectively. From 2001 to 2002 he worked at the University of Waterloo, Canada as a Visiting Scholar. He is currently an associate Professor at the University of Castilla-La Mancha, Ciudad Real, Spain. His research interests include voltage stability, electricity markets and computer-based power system modeling and analysis. 